\title{
Intraoperative radiation therapy for the treatment of recurrent retroperitoneal and pelvic tumors: a single-institution analysis
}

Tharcisio Machado Coelho*, Ricardo César Fogaroli, Antonio Cassio Assis Pellizzon, Douglas Guedes De Castro, Guilherme Rocha Melo Gondim, Maria Leticia Gobo Silva, Michael Jenwei Chen and Henderson Ramos

\begin{abstract}
Background: Patients with recurrent retroperitoneal and pelvic region tumors often require multimodal therapies. Intraoperative radiation therapy (IORT) can deliver high-dose radiation to tumor beds, even if first-line external beam radiation therapy (EBRT) was administered. We evaluated local control (LC) and survival in patients receiving IORT for recurrent tumors.
\end{abstract}

Methods: We retrospectively analyzed 41 patients with isolated pelvic or retroperitoneal recurrences of colorectal, gynecological, or retroperitoneal primary tumors. Following salvage surgery, all patients underwent tumor bed IORT via electron beam or high dose rate brachytherapy. Isolated IORT (median dose: 15 Gy) was administered to patients who had received first-line EBRT; other patients received IORT (median dose 12 Gy) plus EBRT. Local (LF), regional (RF), and distant failures (DF) were evaluated, and the Kaplan-Meier method and log-rank test were used to evaluate and compare overall survival (OS) from the date of IORT.

Results: Forty-one patients underwent 44 treatments, including 27 (61.3\%) isolated IORT and 17 (38.7\%) IORT and EBRT combination regimens. The median follow-up was 8.1 years (range: 4.4-11.7 years), and the 2, 5, and 8 year overall LC rates were $87.9,64.0$, and $49.8 \%$, respectively. Regarding resection status, the respective 2,5 , and 8 year $\mathrm{LC}$ rates were 90,76 , and $76 \%$ for R0 resection and 75, 25, and 0\% for R1 resection $(p<0.001)$. The 2, 5, and 8 year OS rates were 68,43 , and $26 \%$, respectively. OS was better among patients with LC $(p<0.001)$. Twenty-four patients (58.5\%) experienced a DF, and the 5 year OS rates for the patients with and without DF were 36 and 52\%, respectively $(p=0.04)$.

In a multivariate analysis, LF ( $p=0,012)$ and recurrent retroperitoneal sarcoma $(p=0,014)$ were identified as significant predictors of worse OS. Thirteen patients (31\%) developed clinically treatable complications related to IORT.

Conclusions: Many patients achieve long-term OS and LC without significant morbidity after salvage surgery and IORT, especially in case of clear margins.

Keywords: Retroperitoneal and pelvic recurrent tumors, Intraoperative radiation therapy, Local control, Salvage surgery

\footnotetext{
* Correspondence: tharcisiocoelho@hotmail.com

Department of Radiotherapy, A C Camargo Cancer Center, São Paulo, Brazil

(c) The Author(s). 2018 Open Access This article is distributed under the terms of the Creative Commons Attribution 4.0 International License (http://creativecommons.org/licenses/by/4.0/), which permits unrestricted use, distribution, and reproduction in any medium, provided you give appropriate credit to the original author(s) and the source, provide a link to the Creative Commons license, and indicate if changes were made. The Creative Commons Public Domain Dedication waiver (http://creativecommons.org/publicdomain/zero/1.0/) applies to the data made available in this article, unless otherwise stated.
} 


\section{Background}

The curative treatment of retroperitoneal and pelvic primary tumors often involves multidisciplinary approach. However, the local failure (LF) rates for various types of tumors range from 20 to $77 \%[1,2]$, despite treatment regimens comprising surgery, radiotherapy, and/or chemotherapy. Such failures are associated with a worsening quality of life for the patient. Currently, salvage surgery is considered the only curative option for isolated recurrences of retroperitoneal and pelvic tumors (rRPT), especially in patients who have already undergone first-line radiotherapy; however, subsequent failures have been reported in more than $50 \%$ of such cases [3-5]. In other words, surgery alone cannot achieve satisfactory local control (LC). For such cases, adjuvant radiotherapy may reduce LF rates, especially in cases involving positive or close margins [3].

As noted above, treatment options for recurrent disease are limited for patients who have received firstline external beam radiotherapy (EBRT). However, intraoperative radiation therapy (IORT) can be used to administer single high doses of radiation to tumor beds to eliminate microscopic tumor foci while sparing the organs at risk. This modality also increases the likelihood that the tumor bed will be accurately identified without the restrictions of imaging exams $[1,5]$. Furthermore, IORT can be used safely to administer additional doses of radiation to patients previously treated with EBRT [6], thus circumventing the dose limitation imposed by first-line treatment.

To date, few studies have evaluated the safety and efficacy of IORT for the treatment of rRPTs. Therefore, we aimed to evaluate the outcomes of surgery and IORT for rRPT at our institution in terms of LC and survival.

\section{Methods}

\section{Patient and tumor characteristics}

In this retrospective study, we analyzed the medical records of patients who underwent salvage surgery and IORT for isolated rRPTs between June 2004 and April 2015. Patients with metastatic disease or multiple recurrence foci were excluded.

The majority of cases $(92.8 \%)$ were included in three different recurrent tumor groups: Colorectal Tumors, Retroperitoneal Sarcomas, or Gynecological Tumors. Although the patients also included one case each of a retroperitoneal recurrence of pancreatic tumor, pelvic recurrence of a primary soft tissue pelvic sarcoma, and pelvic recurrence of Ewing sarcoma. The cases were also subdivided in two histology: epithelial and sarcoma.

The characteristics of the $41 \mathrm{rRPTs}$ patients who met the above-described criteria and were included in the study are listed in Table 1.
Table 1 Patient characteristics

\begin{tabular}{|c|c|c|}
\hline & $N=41$ & $\%$ \\
\hline \multicolumn{3}{|l|}{ Sex } \\
\hline Female & 27 & $65.8 \%$ \\
\hline Male & 14 & $34.2 \%$ \\
\hline \multicolumn{3}{|l|}{ Age (years) } \\
\hline Median & 51 (range: 18-82) & \\
\hline \multicolumn{3}{|l|}{ Groups stratified by tumor and histology } \\
\hline Gynecologic & 15 & $36.5 \%$ \\
\hline -Uterine endometrioid adenocarcinoma & (4) & \\
\hline -Uterine carcinosarcoma & (2) & \\
\hline -Cervical adenocarcinoma & (4) & \\
\hline -Cervical squamous cell & (4) & \\
\hline -Ovarian adenocarcinoma & (1) & \\
\hline Colorectal & 12 & $29.5 \%$ \\
\hline -Rectal adenocarcinoma & (8) & \\
\hline -Colon adenocarcinoma & (3) & \\
\hline -Anal squamous cell carcinoma & (1) & \\
\hline Retroperitoneal sarcoma & 11 & $26.8 \%$ \\
\hline -Undifferentiated pleomorphic sarcoma & (6) & \\
\hline -Liposarcoma & (4) & \\
\hline -Fibrosarcoma & (1) & \\
\hline Pelvic soft tissue sarcoma & 1 & $2.4 \%$ \\
\hline -Liposarcoma myxoid & $(1)$ & \\
\hline Ewing sarcoma & 1 & $2.4 \%$ \\
\hline -Ewing sarcoma & (1) & \\
\hline Pancreas & 1 & $2.4 \%$ \\
\hline -Adenocarcinoma & (1) & \\
\hline \multicolumn{3}{|l|}{ Tumor histology } \\
\hline Epithelial & 26 & $63.4 \%$ \\
\hline Sarcoma & 15 & $36.6 \%$ \\
\hline
\end{tabular}

\section{Treatment characteristics}

Patients were initially treated via surgery combined with adjuvant or neoadjuvant therapies as appropriate for individual clinical cases. All recurrent cases were discussed in tumor board sessions that comprised the specialists involved with the treatment. When possible and indicated, patients underwent salvage surgery with IORT alone or in combination with EBRT.

IORT was administered via either an electron beam from a Linac linear accelerator, which was directed by cylindrical applicators attached to the collimator, or a high-dose-rate (HDR) brachytherapy source. During surgery, the surgeon and radiation oncologist defined the target volume to be treated (Fig. 1). The anesthetized patients were transported to the Linac/HDR treatment room through an isolated 20-m route. Both the route and treatment room had been subjected to 


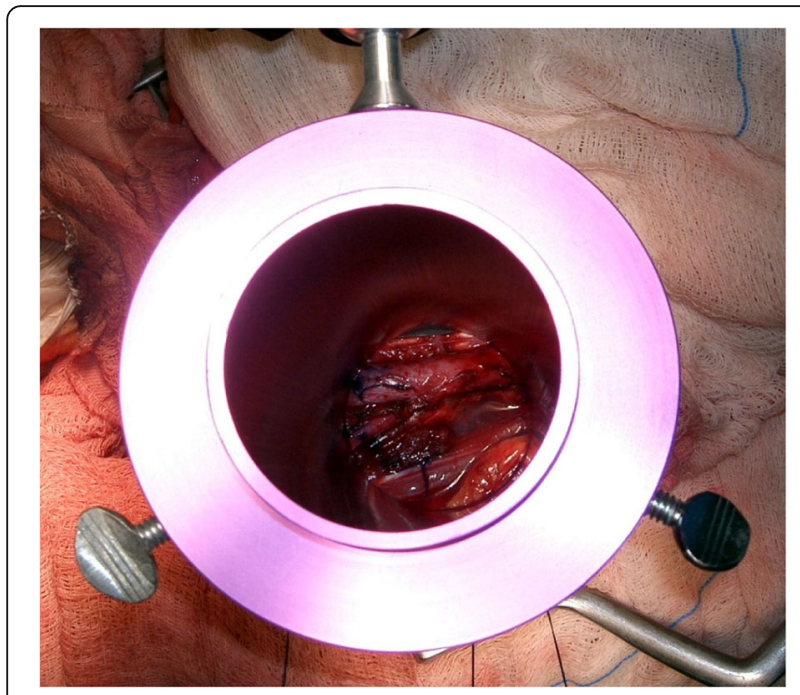

Fig. 1 Delineation of the tumor bed in the pelvic region prior to IOERT

previous terminal cleaning. Cylindrical applicators with variable diameters of 6-12 cm and electron beams of 6-15 MeV or an Iridium $192 \mathrm{HDR}$ source with HAM (Harrison-Anderson-Mick) applicator were used to ensure coverage of the tumor bed with margins of $10 \mathrm{~mm}$. The characteristics of the various treatments are shown in Table 2.

Table 2 Treatment characteristics

\begin{tabular}{|c|c|c|}
\hline & $N=44$ & $\%$ \\
\hline \multicolumn{3}{|l|}{ IORT indication } \\
\hline Pelvic recurrence & 22 & $50 \%$ \\
\hline Retroperitoneal recurrence & 22 & $50 \%$ \\
\hline \multicolumn{3}{|l|}{ Resection } \\
\hline Ro & 36 & $88 \%$ \\
\hline R1 & 08 & $12 \%$ \\
\hline \multicolumn{3}{|l|}{ IORT device } \\
\hline Electron (IOERT) & 43 & $97 \%$ \\
\hline HDR & 01 & $3 \%$ \\
\hline \multicolumn{3}{|l|}{ IOERT dose } \\
\hline Median (first-line EBRT) & 15 Gy (range:10-21 Gy) & \\
\hline Median (no first-line EBRT) & 12 Gy (range: 9-15 Gy) & \\
\hline \multicolumn{3}{|l|}{ IOERT energy } \\
\hline Median & $9 \mathrm{MeV}$ (range: 6-15 MeV) & \\
\hline \multicolumn{3}{|l|}{ Association with adjuvant ebrt } \\
\hline Yes & 17 & $38.6 \%$ \\
\hline No & 27 & $61.4 \%$ \\
\hline \multicolumn{3}{|l|}{ Association with chemotherapy } \\
\hline Yes & 11 & $25 \%$ \\
\hline No & 33 & $75 \%$ \\
\hline
\end{tabular}

Legend: EBRT External beam radiation therapy, IOERT Intraoperative electron radiation therapy
Outcomes of salvage surgical procedures were classified as follows: R0, free surgical margins; R1, resection with focally microscopically involved margins; and R2, visible or palpable residual tumor.

Forty-four treatments were delivered; these included 3 patients who each developed 2 recurrences at different anatomical sites. Only one treatment was performed using HDR, and this case was included in the colorectal tumor group; further, a 12-Gy radiation dose was prescribed to a $5-\mathrm{mm}$ depth from the applicator surface and was associated with non-prior or adjuvant EBRT treatment. Fourteen cases (31.8\%) involved recurrence sites that had been subjected to anterior EBRT at a median dose of 50 Gy (range: $45-60$ Gy). Of these, 8 cases involved rectal tumors that received neoadjuvant treatment at a total dose of 45 Gy in fractions of $1.8 \mathrm{~Gy}$; the first PTV encompassed the tumor and draining lymphatics and received a dose of $45 \mathrm{~Gy}$, whereas the second PTV encompassed the tumor with a 2-cm safety margin in all directions and received a total dose of 50.4 Gy. In four cases involving uterine cervical tumors, the PTV encompassed the tumor bed and draining lymphatics and received an adjuvant dose of 45 Gy in fractions of $1.8 \mathrm{~Gy}$. One case received adjuvant radiation therapy for the treatment of Ewing's sarcoma; a total dose of 55.8 Gy in fractions of 1.8 Gy was delivered to a PTV encompassing the tumor bed with a $2-\mathrm{cm}$ margin. The remaining case involved a pelvic sarcoma and received adjuvant treatment at a total dose of 60 Gy in 2-Gy fractions to a PTV that encompassed the tumor bed with 4-cm margins.

Another 13 cases $(29.5 \%)$ had tumor beds containing a large volume of small intestine and had a high risk of developing actinic enteritis as a complication of EBRT. For these 27 cases, salvage treatment involved surgery and isolated IORT at a median dose of 15 Gy (range: 10-21 Gy). For the other 17 cases (38.6\%), salvage treatments involved a combination of IORT at a median dose of $12 \mathrm{~Gy}$ (range: 9-15 Gy) and an additional adjuvant EBRT course at a total dose of 45 Gy in 25 daily fractions of 1.8 Gy to tumor bed plus 2 to $4 \mathrm{~cm}$ margins in all directions. One patient developed tumor recurrence in the para-aortic lymph node region and received an additional adjuvant course of EBRT to a PTV that encompassed the draining lymphatics in this region. All EBRT treatments realized before 2007 were treated with conformational-technique. From 2007 onwards the treatments were performed using intensity modulated radiotherapy (IMRT). Eleven treatments $(25 \%)$ were administered in association with adjuvant $(n=7)$ or neoadjuvant $(n=4)$ chemotherapy.

\section{Patterns of failure}

An LF was defined as recurrence or tumor progression within the IORT field. Regional failure (RF) was 
defined as recurrence or tumor progression in the retroperitoneal or pelvic region outside of the IORT field. Any other failure was defined as a distant failure (DF). Follow-up evaluations included physical examination, tumor markers, chemistry profiles and IORT anatomical site imaging with CT or MRI. Other imaging tests were ordered depending on patients' clinical complaints. These were scheduled 30 days after IORT and every 3 months until 2 years, every 6 months for 5 years and annually thereafter.

\section{Study endpoints and statistical analysis}

The study endpoints of LF, RF, DF, and overall survival (OS) were evaluated. LF was calculated from the date of IORT to the date of the first in-IORT field recurrence regardless of any previous $\mathrm{DF}, \mathrm{RF}$ was measured from the date of IORT to the date of first outside-IORT field recurrence within the anatomical site(pelvic or retroperitoneal) even in the setting of local recurrence, DF was measured from the date of IORT to the date of first recurrence outside pelvic or retroperitoneal site. OS was calculated according to the Kaplan-Meier method from the date of IORT to the date of death or last contact. Differences in survival outcomes were compared using the log-rank test. The statistical analysis was performed using SPSS, version 24 (IBM, Inc., Armonk, NY, USA). A $p$ value of $\leq 0.05$ was considered statistically significant. Hazard ratio (HR) was used as a summary statistic of censored outcomes. The univariate and multivariate analyses were performed using the Cox regression method to identify risk factors.

Acute and chronic toxicities attributable to IORT were scored according to the RTOG/EORTC Radiation Toxicity Grading System [7] and the National Institutes of Health (NIH) Numeric Rating Scale (NRS-11) [8]. Toxicities attributed to the surgical procedure have been separately described.

\section{Results}

Patients were followed for a median of 8.1 years (range: 4.4-11.7 years).

\section{Local control}

The 2, 5 , and 8 year LC rates were $87.9,64$, and $49.8 \%$, respectively. Figure 2 presents the Kaplan-Meier curve of LC for the entire cohort. In a subgroup analysis, the respective 2, 5, and 8 year LC rates were 90,76 , and $76 \%$ for R0 resection vs. 75,25 , and $0 \%$ for $\mathrm{R} 1$ resection, a significant difference $(p \leq 0.001)$. In summary, the achievement of R0 resection was associated with better LC (Fig. 3). A complete R0 resection was achieved in 36 treatments $(81.8 \%)$, and an $\mathrm{R} 1$ resection was achieved in 8 cases (18.2\%). No procedure yielded an R2 resection.

The 2-, 5-, and 8-year LC rates for colorectal tumors were 71,23 , and $0 \%$, respectively. Among patients with retroperitoneal sarcoma, the 2-, 5-, and 8-year LC rates were 86,62 , and $62 \%$, respectively. The corresponding LC rates for gynecological tumors were 94, 81, and $55 \%$, respectively (Fig. 4). In an analysis stratified

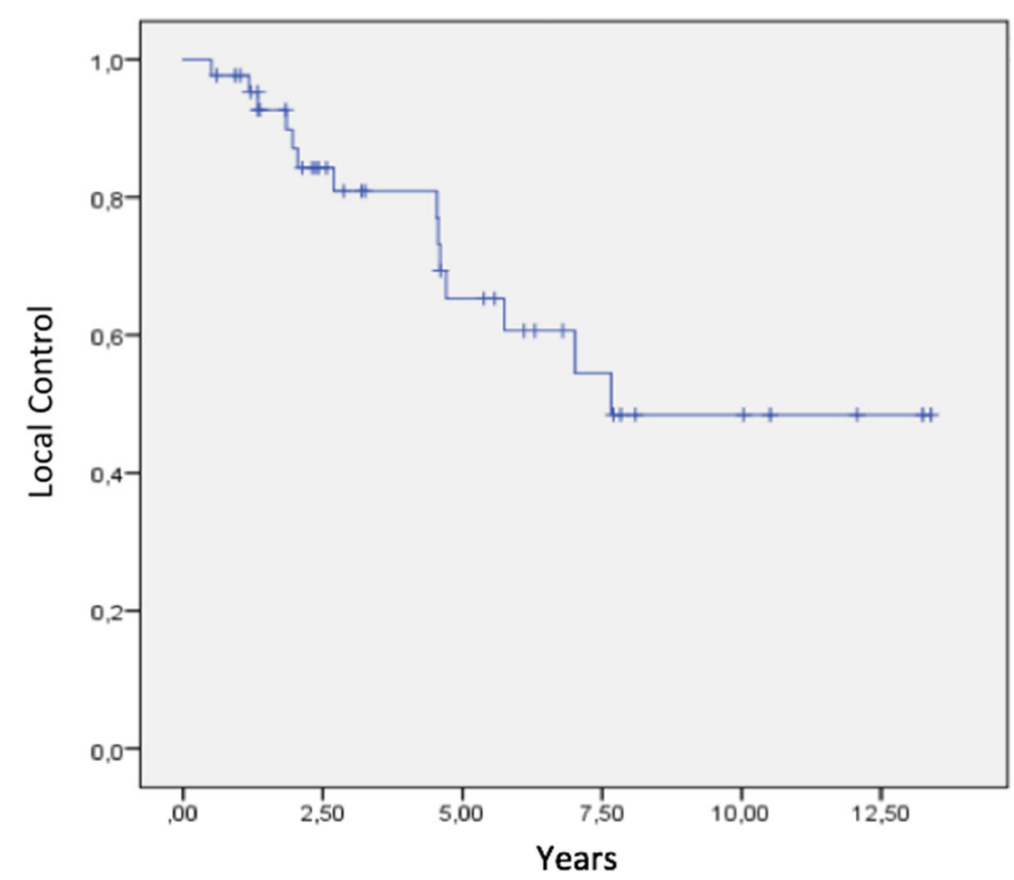

Fig. 2 Local control for the entire cohort 


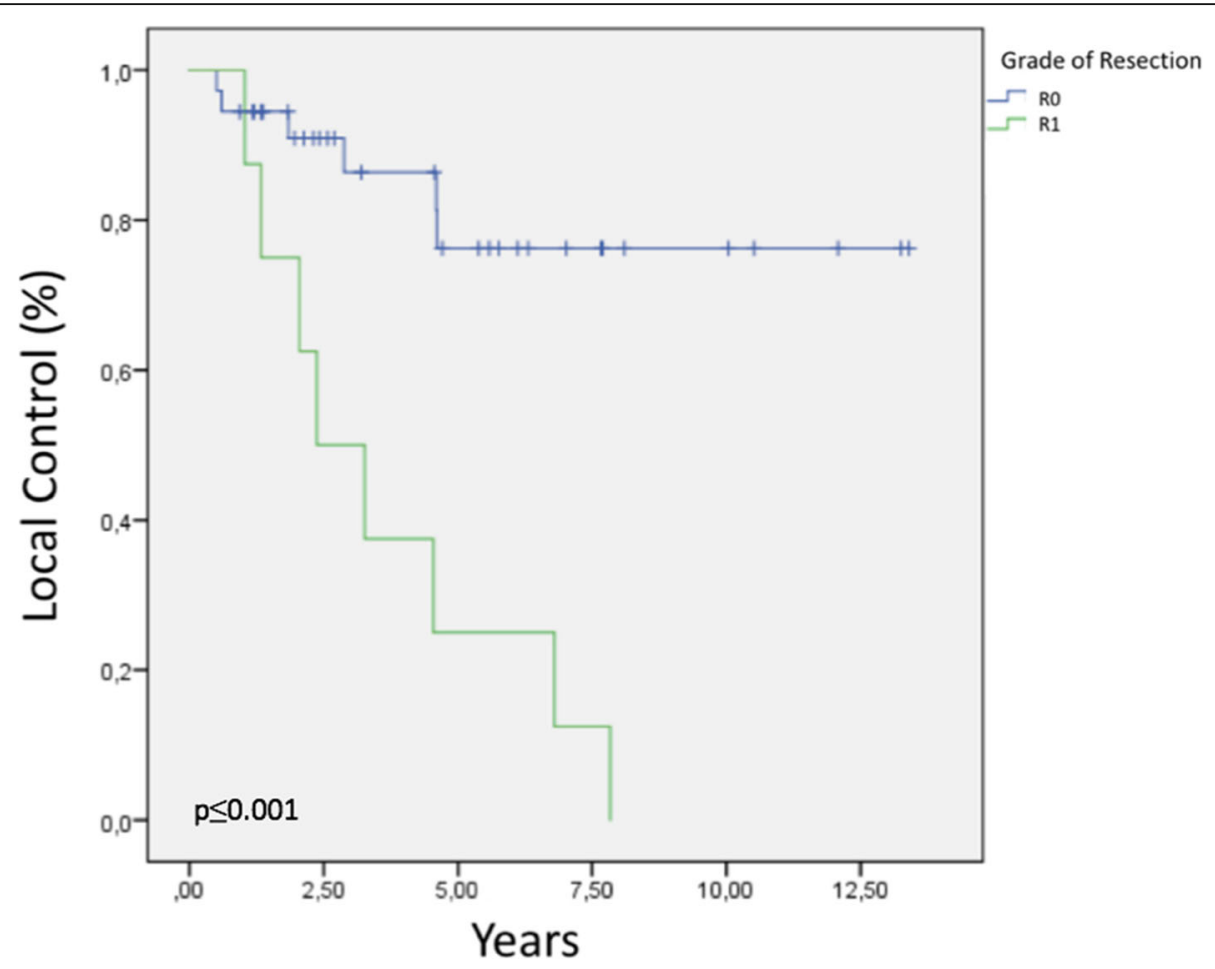

Fig. 3 Local control by resection status

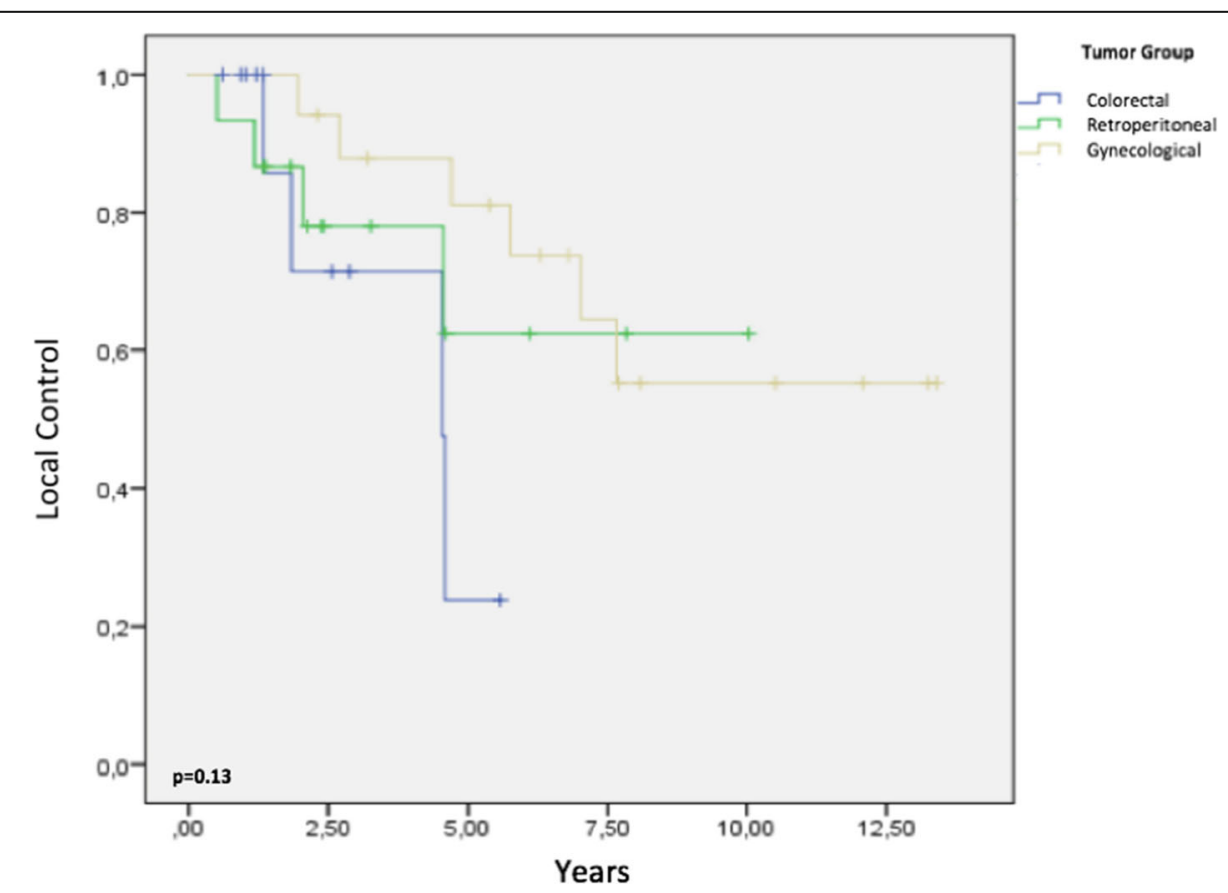

Fig. 4 Local control by tumor group 
according to histology subtypes, the respective 2-, 5-, and 8 -year LC rates were 90,70 , and $47 \%$ for epithelial tumors and 80,58 , and $58 \%$ for sarcomas, respectively $(p=0.56)$.

Treatment-related variables and their effects on the 8-year LC rate are listed under Table 3.

In univariate analysis, only $\mathrm{R} 1$ resection significantly correlated with a high LF rate (HR: 6.7,95\% CI: 2.319.7, $p<0.001)$. In multivariate analysis of the three tumor groups (colorectal, retroperitoneal, and gynecological), a recurrent gynecological tumor was associated with better LC, although this association was only marginally significant (HR: $0.26,95 \%$ CI: $0.06-1.04, p=0.058$ ).

\section{Overall survival}

Among all patients, the 2-, 5-, and 8-year OS rates were 68,43 , and $26 \%$, respectively. The Kaplan-Meier curve of OS for the entire cohort is presented in Fig. 5. Regarding the three tumor groups, the respective 2-, 5 -, and 8 -year OS rates were 83,58 , and $43 \%$ for colorectal tumors $(p=0.09)$; 61,15 , and $7 \%$ for retroperitoneal sarcomas $(p=0.005)$; and 62,56 , and $30 \%$ for gynecological tumors $(p=0.43)$, respectively (Fig. 6). Regarding histology subtypes, the respective 2-, 5-, and 8 -year OS rates were 73,57 , and $40 \%$ for epithelial tumors and 60,20 , and $6 \%$ for sarcomas, respectively $(p=0.005)$.

Table 3 Associations of treatment-related variables with local failure (log-rank)

\begin{tabular}{llll}
\hline Treatment-Related Variables & Category & $\begin{array}{l}\text { Local } \\
\text { Control (8y) }\end{array}$ & $P$ \\
\hline Resection & R0 & $76.2 \%$ & $<0.001$ \\
& R1 & $0 \%$ & \\
EBRT (first treatment) & Yes & $25.9 \%$ & 0.092 \\
& No & $59 \%$ & \\
Association with adjuvant & Yes & $46.9 \%$ & 0.786 \\
EBRT & No & $54.4 \%$ & \\
Association with & Yes & $75 \%$ & 0.854 \\
neoadjuvant chemotherapy & No & $49.5 \%$ & \\
Association with adjuvant & Yes & $62.5 \%$ & 0.950 \\
chemotherapy & No & $45.9 \%$ & \\
Histology & Sarcoma & $58 \%$ & 0.56 \\
& Epithelial & $47 \%$ & \\
Tumor groups & Colorectal & $23 \%(5 y)$, & 0.06 \\
& Tumors & $0 \%(8 y)$ & \\
& Retroperitoneal & $62 \%$ & 0.87 \\
& Sarcomas & & \\
& Gynecological & $55 \%$ & 0.15 \\
& Tumors & & \\
\hline
\end{tabular}

Legend: Treat. Treatment, EBRT External beam radiation therapy
Patients with LF had a significantly worse OS compared with patients with LC (5-year OS: $14 \%$ vs. $59 \%$, $p=0.006)$ (Fig. 7). Six patients (14.6\%) experienced RF and 24 (58.5\%) experienced DF. Patients with and without DF had 5 -year OS rates of 36 and $52 \%$, respectively $(p=0.042)$.

The disease-specific mortality rates were 32,56 , and $71 \%$ at 2,5 , and 8 years, respectively. The treatment-related variables and their effects on OS are presented in Table 4.

In a univariate analysis of the entire cohort, the following prognostic factors were found to correlate with the mortality rate: LF (HR: $2.7,95 \%$ CI: $1.31-5.95, p=$ 0,006 ), absence of DF (HR: 0.43,95\% CI: 0.18-0.99, $p=$ 0.042), recurrent retroperitoneal sarcoma (HR: $2.81,95 \%$ CI $1.33-5.94, p=0.007)$, and sarcoma histology (HR: $2.75,95 \%$ CI 1.31-5.75, $p=0.007$ ).

The results of the univariate analysis are presented in Table 5.

In a multivariate analysis the presence of LF (HR: 2.71, 95\% CI: $1.24-5.92, p=0.012$ ) and retroperitoneal recurrent sarcoma were found to influence the survival rate (HR: 3.40, 95\% CI: $1.27-9.02, p=0.014$ ).

\section{Morbidity}

Sixteen patients experienced toxicities attributable to the surgical procedure, including 3 cases of intestinal obstruction, 3 of lymphocele, 2 of persistent lower limb edema, 2 of ureter stenosis, 2 of operative wound dehiscence, and 1 each of perineal fistula, enteric fistula, pancreatic fistula, and retroperitoneal hematoma. Surgery was required to treat the cases with intestinal obstruction, ureter stenosis, enteric fistula, and operative wound dehiscence. No deaths due to surgical complications were reported. Additionally, 13 patients (31\%) developed complications related to IORT. Seven patients (17\%) developed acute pain with a severity of 4-6 on a 10-point scale that was associated with the procedure and could be controlled with opioids. Two patients (5\%) developed acute grade 2 lower GI enteritis. Chronic toxicity was observed in 3 (7\%) patients with chronic neuralgia controlled with opioids and antidepressants. $1(2,5 \%)$ patient developed ischiatic osteomyelitis 1 year after IORT. No deaths were attributed to toxicities.

\section{Discussion}

This report describes our single-center experience with a multimodality approach comprising salvage surgery and IORT for the treatment of rRPTs. Notably, our analysis demonstrated satisfactory long-term LC rates, particularly for cases in which an R0 resection was achieved. 


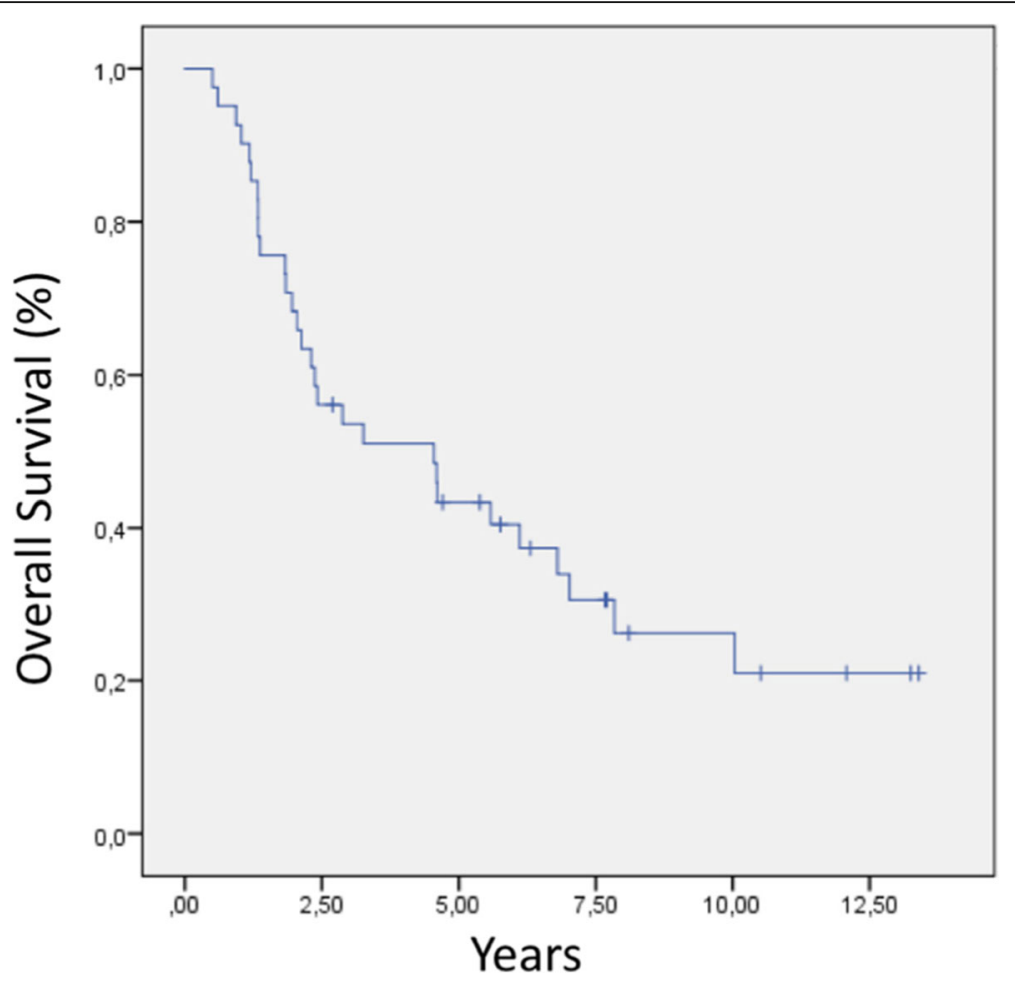

Fig. 5 Overall survival for the entire cohort

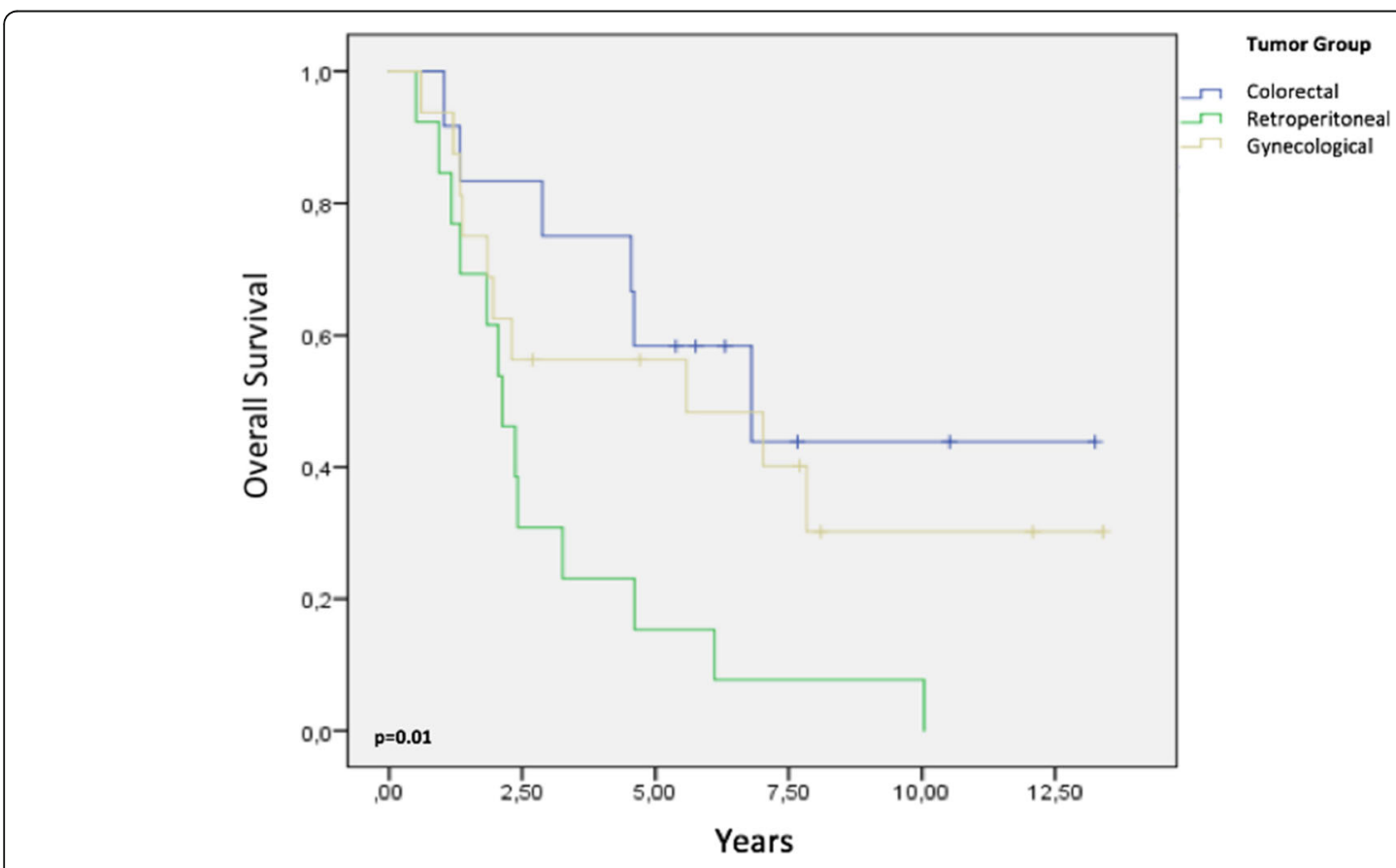

Fig. 6 Survival analysis by tumor group 


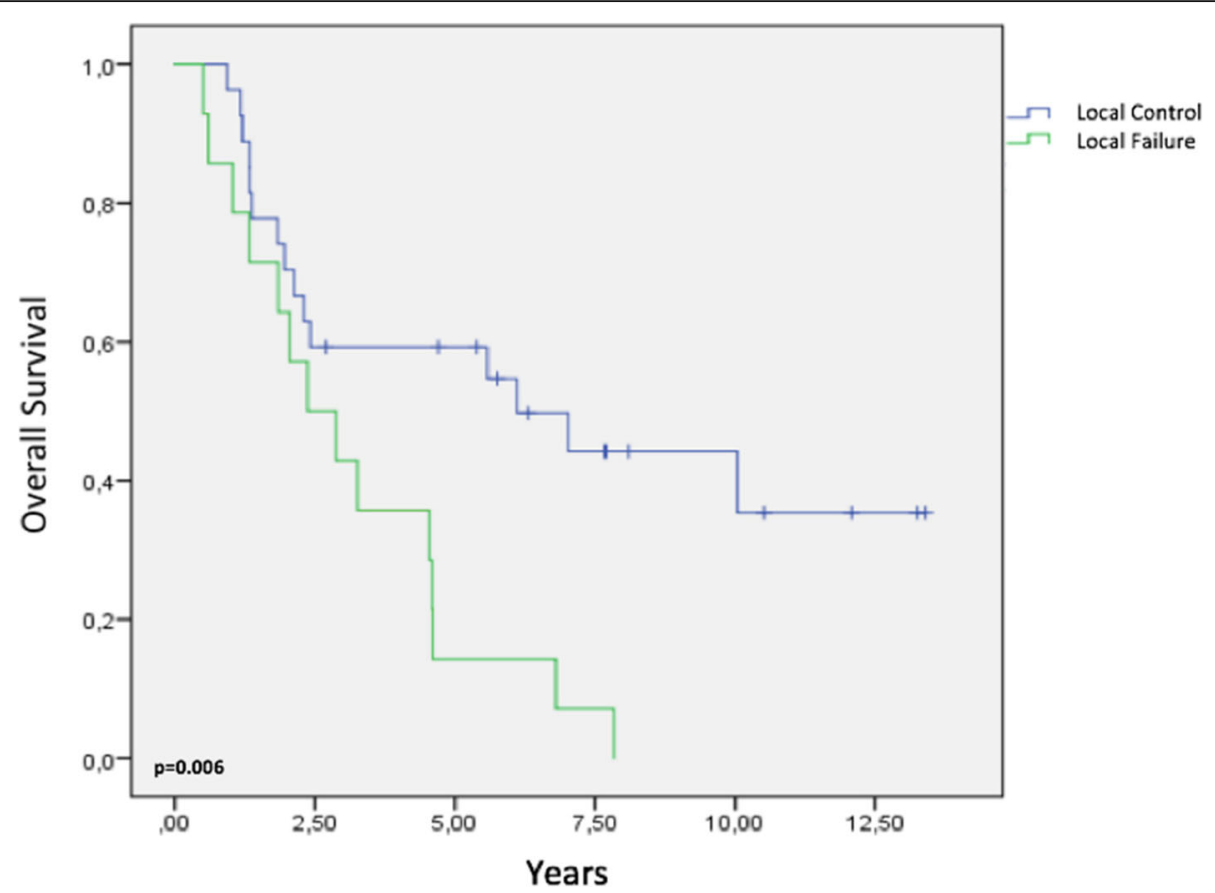

Fig. 7 Survival analysis by local control status

Our study included several histological tumor types and thus demonstrated the different clinical scenarios wherein IORT can be employed. In a study involving 128 patients, Cambeiro et al. [9] also analyzed the results of IORT for the treatment of several types of tumors, such as soft tissue sarcomas as well as head and neck, uterine, and colorectal tumors. The authors used a treatment regimen similar to that used in our study, with association of EBRT (median dose 46 Gy) and IORT (median dose 15 Gy) in $58 \%$ of the cases. In a multivariate analysis, only the degree of resection statistically significantly influenced LC, such that the patients who underwent $\mathrm{R} 2$ resection had a 2.2 -fold higher risk of treatment failure (95\% CI: $1.2-4.1 ; p=$ 0.007). In that study, patients with histologically epithelial tumors had lower survival rates. By contrast, in our study, we observed worse survival outcomes associated with sarcoma histology, which we attribute to the presence of this histology in tumors with more aggressive clinical behavior, such as recurrent retroperitoneal and uterine sarcomas. In relation to dose intensity, the authors concluded that the treatments performed with a combination of IOERT and EBRT, with a median value for tumor control standardized to 2 Gy equivalents (EQD2) $\geq 62$ Gy had a statistically significant influence on overall survival, compared to treatments with isolated IOERT, with a median value of EQD2 of 31.2 Gy (HR 2.2, 95\% CI: 1.1-4.1). In our study, the median value of the EQD2 for the treatments performed with the association of the two modalities and with isolated IORT were $66.2 \mathrm{~Gy}$ and 31.2 Gy respectively, but we did not verify influence on overall survival, probably due to the restricted number of patients and by the limitations of a retrospective study.

Below, we further discuss the outcomes of our study in terms of the type of recurrence.

\section{Recurrent colorectal tumors}

Recurrent colorectal cancers present unique challenges in terms of management, as previous EBRT limits the available options for further radiation treatment. However, IORT may be indicated in select cases.

Previous studies of IORT have reported variable LC and OS outcomes. One extensive IORT review demonstrated a significant improvement in LC among patients with locally recurrent pelvic tumors [10]. Furthermore, in a meta-analysis of $>3000$ patients, Mirnezami et al. observed benefits with IORT in terms of LC, disease-free survival, and OS among patients with recurrent rectal and advanced colorectal cancers [11]. A Cleveland Clinic study reported a 1 year LF rate of $16 \%$ and 3 year OS rate $49 \%$ among patients who underwent IORT for locally advanced and recurrent rectal cancers [12], and Roeder et al. reported 5 year LF and OS rates of 59 and 30\%, respectively, in a similar clinical situation [3]. Hyngstrom et al. reported 1,3 , and 5 year LF rates of 16,40 , and $44 \%$, respectively, with high-dose-rate intraoperative brachytherapy [13].

In one case involving a recurrent colorectal tumor in the lateral pelvic wall, we performed intraoperative 
Table 4 Associations of treatment-related variables with overall survival after 8 years (log-rank)

\begin{tabular}{|c|c|c|c|}
\hline Treatment-Related Variables & Category & $\begin{array}{l}\text { Overall } \\
\text { Survival }\end{array}$ & $P$ \\
\hline \multirow[t]{2}{*}{ Sex } & Male & $28.6 \%$ & 0.876 \\
\hline & Female & $25.5 \%$ & \\
\hline \multirow[t]{2}{*}{ Age (years) } & $<51$ & $30.6 \%$ & 0.243 \\
\hline & $>51$ & $23.5 \%$ & \\
\hline \multirow[t]{2}{*}{ Region of recurrence } & Pelvic & $20.8 \%$ & 0.588 \\
\hline & Retroperitoneal & $33.4 \%$ & \\
\hline \multirow[t]{2}{*}{ Local failure } & Yes & $44.2 \%$ & 0.006 \\
\hline & No & $0 \%$ & \\
\hline \multirow[t]{2}{*}{ Distant failure } & Yes & $10.3 \%$ & 0.042 \\
\hline & No & $52.9 \%$ & \\
\hline \multirow[t]{2}{*}{ Resection grade } & RO & $35.8 \%$ & 0.12 \\
\hline & R1 & $0 \%$ & \\
\hline \multirow[t]{2}{*}{ EBRT (first-line treatment) } & Yes & $17.9 \%$ & 0.109 \\
\hline & No & $30.4 \%$ & \\
\hline \multirow{2}{*}{$\begin{array}{l}\text { Association with adjuvant } \\
\text { EBRT }\end{array}$} & Yes & $21.4 \%$ & 0.687 \\
\hline & No & $34.7 \%$ & \\
\hline \multirow{2}{*}{$\begin{array}{l}\text { Association with neoadjuvant } \\
\text { chemotherapy }\end{array}$} & Yes & $0 \%$ & 0.157 \\
\hline & No & $28.6 \%$ & \\
\hline \multirow{2}{*}{$\begin{array}{l}\text { Association with adjuvant } \\
\text { chemotherapy }\end{array}$} & Yes & $38.1 \%$ & 0.347 \\
\hline & No & $23.1 \%$ & \\
\hline \multirow[t]{2}{*}{ Histology } & Sarcoma & $6,7 \%$ & 0.005 \\
\hline & Epithelial & $40 \%$ & \\
\hline \multirow[t]{3}{*}{ Group of tumors } & $\begin{array}{l}\text { Colorectal } \\
\text { Tumors }\end{array}$ & $43 \%$ & 0.09 \\
\hline & $\begin{array}{l}\text { Retroperitoneal } \\
\text { Sarcomas }\end{array}$ & $7 \%$ & 0.005 \\
\hline & $\begin{array}{l}\text { Gynecological } \\
\text { Tumors }\end{array}$ & $30 \%$ & 0.43 \\
\hline
\end{tabular}

Legend: EBRT External beam radiation therapy

brachytherapy using a HAM applicator because the anatomical tumor bed location did not allow adequate electron-beam therapy.

Several previous studies have identified surgical resection margin status as an important factor affecting LC during treatment of patients with local recurrences of colorectal cancer. In a recent clinical review, Haddock MG [14] extensively analyzed several studies and demonstrated that in selected series involving IORT for the treatment of recurrent rectal cancer, the $\mathrm{LC}$ rates were $60-80 \%$ and the 5 -year survival rates were $40-50 \%$. Among cases with microscopically positive margins, the LC rates ranged from 30 to $60 \%$ and the 5 -year survival rates ranged from 20 to $30 \%$. Other studies have also evaluated the influence of resection grade on the LC of recurrent colorectal tumors. For example, in a study by Roeder et al., patients with a
Table 5 Results of a univariate analysis of the associations of various clinical characteristics with overall survival

\begin{tabular}{lll}
\hline Clinical Characteristic & Category & $\begin{array}{l}\text { HR (Univariate) } \\
95 \% \mathrm{Cl}\end{array}$ \\
\hline Sex & Male & $1.06(0.48-2.36)$
\end{tabular}

Age ( $\geq$ vs. $<51$ years)

Female (Ref.)

$<51$ years

$1.57(0.72-3.42)$

$\geq 51$ years (Ref.)

Region of recurrence Pelvic

$0.81(0.38-1.70)$

Retroperitoneal (Ref.)

Local failure

No

$2.79(1.31-5.95)$

Yes (Ref.)

Resection grade $\quad$ RO

$1.87(0.82-4.27)$

R1 (Ref.)

Association with

first-line EBRT

Association with adjuvant EBRT

Association with

neoadjuvant chemotherapy

Association with adjuvant chemotherapy

Yes

$0.53(0.24-1.16)$

No (Ref.)

Yes

$1.16(0.55-2.45)$

No (Ref.)

Yes

No (Ref.)

Yes

No (Ref.)

Distant failure

Yes

No (Ref.)

Histology

Sarcoma (Ref.)

$2.75(1.31-5.75)$

Epithelial

Colorectal Tumor

Yes (Ref.)

$0.47(0.19-1.16)$

No

Retroperitoneal Sarcoma

Yes (Ref.)

$2.81(1.33-5.94)$

No

Gynecological Tumor

Yes (Ref.)

$0.73(0.34-1.58)$

No

status of R0 after the resection of locally recurrent rectal cancer were found to have 5 year LC and OS rates that were respectively threefold and fivefold better than those of patients with incomplete resections [3]. Consistent with that report, three resections of recurrent rectal tumors (25\%) in our series achieved an R1 status, and none obtained LC. Holman et al. reported a 5 year LF of $45 \%$ in a pooled analysis of 565 patients treated with IORT for advanced and recurrent rectal cancers and identified the resection grade as a risk factor for LC [15]. Dresen et al. [16] also observed worse LC rates following $\mathrm{R} 1$ resection in an analysis of 147 patients with local recurrences of rectal cancer. In his series, the median OS duration was 28 months, whereas the 5-year OS, DFS, metastasis-free survival, and LC rates were $31.5,34.1,49.5$, and $54.1 \%$, 
respectively. Resections $\mathrm{R} 0, \mathrm{R} 1$ and $\mathrm{R} 2$ was achieved in 84 (57.2\%), 34 (23.1\%), and 29 (19.7\%) patients respectively. For patients with a resection R0, median Os was 59 months and 5-years OS and LC were $48,4 \%$ and $68,9 \%$ respectively $(p<0,001)$. In our study, these treatments yielded 9 R0 (75\%) and 3 R1 resections (25\%), with a 5 year LC rate of $73 \%$. Consistent with that report results, none of the resections with $\mathrm{R} 1$ status obtained local control.

Despite the favorable LC and OS outcomes of IORT, treatment complications are reported with relative frequency in the literature. In our experience, we observed chronic neuralgia in 3 patients and osteomyelitis in 1 patient. Suzuki et al. reported a 5 year LC rate of $60 \%$ among patients who received EBRT before or after adjuvant IORT [17]; however, this outcome was unfortunately accompanied by a grade 3 toxicity incidence exceeding $30 \%$. Similarly, Willet et al. reported a complication rate of $30 \%$ after preoperative EBRT associated with IORT for recurrent pelvic tumors [18]; here, most events involved soft tissue or sacral injury and pelvic neuropathy. Roeder et al. identified wound healing disturbance as the most common complication (20\% of patients), followed by abscess or fistula formation (16\%) and severe chronic pain (8\%) [3].

Finally, most relevant literature reports have reported DF with this type of neoplasia. Consistent with those reports, half of the 12 cases of recurrent colorectal tumors in our study developed a DF, including 6 cases of liver metastasis.

\section{Recurrent retroperitoneal sarcomas}

Approximately $38 \%$ of all sarcomas arise in the retroperitoneum [19], and surgery is currently the main treatment with curative intent. In contrast to sarcomas of the extremities, however, wide surgical margins are often not achievable in the retroperitoneum; accordingly, local progression is the dominant pattern of failure. As the retroperitoneum contains many critical organs with low radiation tolerances, adequate dose delivery via EBRT either cannot be achieved or would result in excessive toxicity. By contrast, IORT facilitates the delivery of a single high radiation dose to the tumor bed during surgery while sparing the surrounding organs at risk via physical distance from the radiation field or adequate lead shielding. The total combined dose from IORT plus moderate EBRT increases the possibility of LC while reducing toxicity.

Regional failure is commonly observed among patients with retroperitoneal sarcoma, including 5 patients in our study; an additional 5 patients experienced DF.

No randomized data regarding radiotherapy for retroperitoneal sarcoma are currently available. An ongoing EORTC study (NCT01344018), Surgery with or without Radiation Therapy in Untreated Nonmetastatic Retroperitoneal Sarcoma (STRASS), is currently recruiting participants. Furthermore, propensity score-matched analyses of more than 9000 patients with resected retroperitoneal sarcoma with or without preoperative or postoperative irradiation in the National Cancer Data Base demonstrated improved median OS in the irradiation group compared with the no-irradiation group, regardless of the irradiation time (110 months for preoperative vs. 89 months for postoperative vs. 66 months for no irradiation) [20].

Regarding LC, other authors have reported results similar to ours. Petersen et al. evaluated the management of 44 cases of recurrent retroperitoneal soft tissue sarcoma treated with IORT at the Mayo Clinic; the reported LF rate of $39 \%$ was comparable with our series. The authors also reported a 5 year OS rate of $48 \%$, with no differences were found by primary or recurrent tumor status [21].

Hager et al. compared two groups of patients; although all underwent surgery, half also received radiation therapy [22]. As in our study, Hager and colleagues typically administered a median IORT dose of 15 Gy using an electron energy of $6 \mathrm{MeV}$. The combination of surgery and IORT significantly improved survival outcomes, compared with surgery alone $(p=0.04)$. For all patients, resections R1 and $\mathrm{R} 2$ resection have a decreasing in 5 years survival rate by $7.6 \%$ and by $34.7 \%$ respectively compared to R0 resection.

Recently, Roeder et al. [23] published a retrospective study involving 156 patients among whom 87 had recurrent tumors. Total 114 patients were treated with a combination of IORT (median dose: 15 Gy) and EBRT (median dose: 45 Gy). During a median follow-up of 38 months, the LC rates at 3 and 5 years were 57 and $50 \%$, respectively. In a multivariate analysis, the tumor grade resection margins and the association with EBRT remained statistically significant.

\section{Recurrent gynecological tumors}

Patients with recurrent gynecological tumors (e.g., tumors of the uterine cervix, endometrium, and ovary) often present with lesions on the pelvic walls and/or involvement of the pelvic or paraaortic lymph nodes. Again, a previous treatment history of high-dose EBRT for these tumors limits the options for salvage radiation therapy. Accordingly, IORT is an important therapeutic option.

IORT has been studied for the treatment of recurrent gynecologic tumors since the 1990s. In a Mayo Clinic study of 148 patients, 125 patients had recurrent gynecological tumors and 113 received IORT associated with EBRT [24]. In that study, the 5 year LF rate was $40 \%$ and the 5 years OS was 27\%. Furthermore, R2 resection was associated with a worse 5 year OS rate, compared with $\mathrm{R} 0$ or $\mathrm{R} 1$ resection ( $31 \%$ vs $13 \%, p=0.01$ ).

Tumors of the uterine cervix are common in Brazil and are often diagnosed at advanced stages. Some retrospective studies in the literature have evaluated the use of IORT 
for the treatment of recurrent uterine cervical cancer. Tran et al. evaluated 17 patients treated with orthovoltage IORT at a median dose of 11.5 Gy [25]. In that study, the LC, metastasis-free survival, and specific survival rates were 45,60 , and $46 \%$, respectively. Similar to our treatment scheme, a Spanish study reported the outcomes of 36 patients with recurrent primary cervical tumors who were treated with IORT (median dose: 15 Gy) alone or combined with EBRT (45 Gy at 1.8 Gy per fraction) if prior radiotherapy had not been administered [26]. In that study, the 10 year LC was $47 \%$, and factors that adversely affected LC included parametrial margin involvement, R1 resection, and pelvic lymph node involvement.

The Mayo Clinic reported the outcomes of 25 patients treated with IORT for recurrent endometrial cancer [27]. Most patients presented with involvement of the pelvic sidewall or paraaortic nodes. In that study, 21 of 25 patients received EBRT (median dose: 45 Gy). The median IORT dose of 15 Gy was consistent with our study. The median survival duration was 57 months, and the 5 year OS rate was $47 \%$. The authors of that study reported that the resection grade influenced survival, with 5 year OS rates of 71,40 , and $0 \%$ among $\mathrm{R} 0, \mathrm{R} 1$, and $\mathrm{R} 2$ cases, respectively. LF within the IORT field and DF were observed in $4(16 \%)$ and 6 patients (24\%), respectively. Given the small number of patients with recurrent gynecological tumors in our study, we could not analyze the influence of histological type on survival. However, other studies have reported better survival outcomes for recurrent endometrial tumors treated with IORT. In a study involving 36 patients with recurrent gynecological malignancies conducted by Arias et al. [28], an endometrial histology was found to correlate with better rates of local PFS ( $p=$ $0.017)$ and OS $(p=0.038)$. Furthermore, older patients exhibited significantly better distant PFS outcomes ( $p=$ 0.015), and patients with endometrial cancer tended to better distant PFS relative to patients with recurrent cervical and vulvar tumors.

Investigators at Stanford University reported the use of orthovoltage IORT to treat 22 patients with recurrent ovarian cancer [29]. In that study, a median dose of 12 Gy was administered to various sites, including the pelvis, paraaortic nodes, inguinal nodes, and porta hepatis. Nine, 5 , and 6 patients received whole-abdominal EBRT, loco-regional EBRT, and associated chemotherapy, respectively. The median survival duration was 26 months, and the 5 year OS and disease-free survival rates were 22 and $18 \%$, respectively. LC and RC was $68 \%$ at 22 months. Furthermore, $55 \%$ of patients experienced DF; consistent with our study in which 9 patients $(60 \%)$ experienced DF, the authors identified this failure pattern as an important influence on OS.

For the various types of tumors, we observed a direct influence of the surgical margin status on LC. Some authors believe that $\mathrm{R} 1$ or $\mathrm{R} 2$ resection may be a consequence of selection of tumor variants with more aggressive biological behaviors following the initial course of radiotherapy and chemotherapy [16]. Despite progress in the quality and precision of imaging exams and the rigorous selection of clinical cases, a true evaluation of the possibility of obtaining a R0 surgical margin may only be possible during surgery. In our experience, we considered cases with increased risk and those with tumors very close to bone structures, such as recurrences of pelvic tumors in the pre-sacral space, recurrences of retroperitoneal sarcomas in the paravertebral region, and lesions adhered to vascular structures. Such cases may be considered "borderline" and should be the subject of a more extensive evaluation to determine the indications and contraindications for IORT. The intraoperative identification of R1 or R2 margins might indicate the need for a dose increase, which is often difficult to accommodate depending on the previously administered EBRT dose and the presence of high-risk anatomical structures in the tumor bed. Other alternative treatment techniques for recurrences of pelvic tumors have been reported in the literature. Murray et al. [30] analyzed 17 studies of stereotactic ablative radiotherapy (SART) for the treatment of 205 patients with recurrent malignant disease within the pelvis. SART has the advantage of allowing the previous treatment planning, optimizing the coverage of the target volume of treatment. The treatment being performed in a fractionated dose regimen allows the radiobiological advantage of better protection of normal structures.

The authors reported 1-year CL indices ranging from 51 to $100 \%$ and a low rate of grade 3 or 4 complications.

\section{Morbidity}

Surgeries performed for the salvage treatment of pelvic or retroperitoneal tumor recurrences are extensive, debilitating, and potentially complicated by previous treatments. First-line EBRT induces fibrosis in tissues surrounding the recurrent tumor and increases the difficulty of the surgical procedure. We observed some serious complications; however, they were fortunately not fatal. Of these complications, $70 \%$ occurred within 90 days of surgery and could be attributed to the surgical procedure.

Peripheral neuropathy is the most commonly reported toxicity attributed to pelvic IORT. IORT-related neuropathies usually present with pain but no significant motor or sensory loss. The pain is usually chronic, possibly severe, and is often manageable with analgesics, including opioids. In our study, this complication occurred in 7 patients and mainly affected those who received treatment for recurrent pelvic region tumors (5 cases). In such cases, pain was likely attributable to the presence of nerve structures in the treatment field (i.e., very near the tumor bed); here, nerves cannot be shielded and thus receive the full IORT dose. 
Some authors have attempted to correlate the incidence of neuropathy with the IORT dose. A Mayo Clinic analysis involving 51 patients treated with IORT (doses: 10-25 Gy) in association with EBRT for primary or recurrent pelvic tumors observed grade 1-3 neuropathy in $32 \%$ of patients [31]. Haddock et al. analyzed the relationship between the IORT dose and incidence of neuropathy in patients receiving treatment for recurrent rectal tumors and noted that IORT doses of $\geq 12.5$ Gy were associated with an incidence of $5 \%$ grade 2 or 3 neuropathy, whereas doses $>15$ Gy were associated with $14 \%$ incidence of grade 2 or 3 neuropathy [32]. Of the 7 patients who developed pain in our study, 6 received an IORT dose $\geq 15$ Gy (range: 15-20 Gy). However, given the small number of patients with this complication, it was not possible to establish a statistical association between the IORT dose and the incidence of neuropathy.

\section{Conclusions}

In this study, a rigorous adherence to follow-up, including accurate imaging studies, facilitated the detection of isolated recurrent retroperitoneal and pelvic tumors. For selected cases, salvage treatment comprising surgery and IORT, either alone or with EBRT, yielded satisfactory LC and survival outcomes with acceptable morbidity. Despite this favorable LC outcome, however, survival was strongly influenced by the occurrence of DF. Our findings underscore the importance of individualized case discussions by tumor boards, as well as the need for additional studies to identify more effective and targeted systemic treatments for this specific group of patients.

\section{Abbreviations}

Cl: Confidence interval; DF: Distant failure; EBRT: External beam radiation therapy; HDR: High dose rate; HR: Hazard ratio; IOERT: Intraoperative electron radiation therapy; IORT: Intraoperative radiation therapy; LC: Local control;

LF: Local failure; OS: Overall survival; PTV: Planning target volume; RF: Regional failure

\section{Acknowledgements}

Not applicable.

\section{Funding}

Not applicable.

Availability of data and materials

Not applicable.

\section{Authors' contributions}

CTM and FRC collected patient data and drafted the article. PACA, CDG, and GGRM collaborated in the discussion. SMLG, CMJ, and RH participated in tumor board meetings and clinical case selection. All authors attended the follow-up visits and read and approved the final manuscript.

\section{Ethics approval and consent to participate}

This was a retrospective study and all data were collected according to the guidelines of the Ethics Committee in Medical Research of the AC Camargo Cancer Center.
Consent for publication

Not applicable.

\section{Competing interests}

The authors declare that they have no competing interests.

\section{Publisher's Note}

Springer Nature remains neutral with regard to jurisdictional claims in published maps and institutional affiliations.

Received: 16 April 2018 Accepted: 30 October 2018

Published online: 20 November 2018

\section{References}

1. Palta M, Willett CG, Czito BG. Cancer of the colon and rectum. In: Halperin EC, Wazer DE, Perez CA, Brady LW, editors. Perez and Brady's Principles and Practice of Radiation Oncology.6rd ed. Philadelphia: Lippincott Williams \& Wilkins; 2013. p. 1229-30.

2. Buchsbaum JC, Douglas JG, Dasgupta B, Lawenda BD. Retroperitoneal Cancer. In: Halperin EC, Wazer DE, Perez CA, Brady LW.Perez and Brady's Principles and Practice of Radiation Oncology.6rd ed. Philadelphia: Lippincott Williams \& Wilkins; 2013. p.1519-1520.

3. Roeder F, Goetz JM, Habl G, Bischof M, Krempien R, Buechler MW, et al. Intraoperative Electron radiation therapy (IOERT) in the management of locally recurrent rectal cancer. BMC Cancer. 2012;12:592.

4. Saito N, Koda K, Takiguchi N, Oda K, Ono M, Sugito M. Curative surgery for local pelvic recurrence of rectal cancer. Dig Surg. 2003;20:192-9.

5. Torres MA, Ballo MT, Butler CE, Feig BW, Cormier JN, Lewis VO, et al. Management of locally recurrent soft-tissue sarcoma after prior surgery and radiation therapy. Int J Radiat Oncol Biol Phys. 2007;67:1124-9.

6. Debenham BJ, Hu KS, Harrison LB. Present status and future directions of intraoperative radiotherapy. Lancet Oncol. 2013;14:e457-64.

7. Cox JD, Stetz J, Pajak TF. Toxicity criteria of the radiation therapy oncology group (RTOG) and the European organization for research and treatment of cancer (EORTC). Int J Radiation Oncol Biol Phys. 1995;31:1341-6.

8. Frattali CM. National Institutes of Health, Warren Grant Magnuson Clinical Center. ASHA. 1999:41:46.

9. Cambeiro M, Calvo FA, Aristu JJ, Jimenez MM, San-Julian M, Alacide J, et al. Salvage surgery and radiotherapy including intraoperative electron radiotherapy in isolated locally recurrent tumors: predictors of outcome. Radiother Oncol. 2015;116:316-22.

10. Pilar A, Gupta M, Laskar SG, Laskar S. Intraoperative radiotherapy: review of techniques and results. Ecancermedicalscience. 2017;11:750.

11. Mirnezami R, Chang GJ, Das P, Chandrakumaran K, Tekkis P, Darzi A, et al. Intraoperative radiotherapy in colorectal cancer: systematic review and meta-analysis of techniques, long-term outcomes, and complications. Surg Oncol. 2013;22:23-35.

12. Mathis $\mathrm{KL}$, Nelson H, Pemberton JH, Haddock MG, Gunderson LL. Unresectable colorectal cancer can be cured with multimodality therapy. Ann Surg. 2008:248:592-8.

13. Hyngstrom JR, Tzeng CW, Beddar S, Das P, Krishnan S, Delclos ME, et al. Intraoperative radiation therapy for locally advanced primary and recurrent colorectal cancer: ten-year institutional experience. J Surg Oncol. 2014;109: $652-8$.

14. Haddock MG, Gunderson LL, Nelson H, Cha SS, Devine RM, et al. Intraoperative irradiation for locally recurrent colorectal cancer in previously irradiated patients. Int J Radiat Oncol Biol Phys. 2001;49:1267-74.

15. Holman FA, Haddock MG, Gunderson LL, Kusters M. Results of intraoperative electron beam radiotherapy containing multimodality treatment for locally unresectable T4 rectal cancer: a pooled analysis of the Mayo Clinic Rochester and Catharina Hospital Eindhoven. J Gastrointest Oncol. 2016;7:903-16.

16. Dresen RC, Gosens MJ, Martijn H, Nieuwenhuijzen GA, Creemers GJ, DanielsGooszen AW, et al. Radical resection after IORT-containing multimodality treatment is the most important determinant for outcome in patients treated for locally recurrent rectal cancer. Ann Surg Oncol. 2008;7:1937-47.

17. Suzuki K, Devine RM, Dozois RR, Gunderson LL, Martenson JA, Weaver AL, et al. Intraoperative irradiation after palliative surgery for locally recurrent rectal cancer or rectosigmoid carcinoma. Cancer. 1995:75:939-52.

18. Willet CG, Shellito PC, Tepper JE, Eliseo R, Convery K, Wood WC. Intraoperative electron beam radiation therapy for recurrent locally advanced rectal or rectosigmoid carcinoma. Cancer. 1991;67:1504-8. 
19. Brennan MF, Antonescu CR, Moraco N, Singer S. Lessons learned from the study of 10,000 patients with soft tissue sarcoma. Ann Surg. 2014;260:416-21.

20. Nussbaum DP, Rushing CN, Lane WO, Cardona DM, Kirsch DG, Peterson BL, et al. Preoperative or postoperative radiotherapy versus surgery alone for retroperitoneal sarcoma: a case control, propensity score-matched analysis of a nationwide clinical oncology database. Lancet Oncol. 2016;17:966-75.

21. Petersen IA, Haddock MG, Donohue JH, Nagorney DM, Grill JP, Sargent DJ, et al. Use of intraoperartive electron beam radiotherapy in the management of retroperitoneal soft tissue sarcomas. Int J Radiat Oncol Biol Phys. 2002:52:469-75.

22. Hager S, Makowiec F, Henne K, Hopt UT, Wittel UA. Significant benefits in survival by the use of surgery combined with radiotherapy for retroperitoneal soft tissue sarcoma. Radiat Oncol. 2017;12:29.

23. Roeder F, Alldinger I, Uhl M, Saleh-Ebrahimi L, Schimmack S, Mechtersheimer $\mathrm{G}$, et al. Intraoperative electron radiation therapy in retroperitoneal sarcoma. Int J Radiation Oncol Biol Phys. 2018;100:516-27.

24. Haddock MG, Petersen IA, Webb MJ, Wilson TO, Podratz KC, Gunderson LL IORT for locally advanced gynecological malignancies. Front Radiat Ther Oncol. 1997;31:256-9.

25. Tran PT, Su Z, Hara W, Husain A, Teng N, Kapp DS. Long-term survivors using intraoperative radiotherapy for recurrent gynecologic malignancies. Int J Radiat Oncol Biol Phys. 2007;69:504-11.

26. Martínez-Monge R, Jurado M, Aristu JJ, Moreno M, Cambeiro M, Pe A, et al. Intraoperative electron beam radiotherapy during radical surgery for locally advanced and recurrent cervical cancer. Gynecol Oncol. 2001;82:538-43.

27. Dowdy SC, Mariani A, Cliby WA, Haddock MG, Petersen IA, Sim FH, et al. Radical pelvic resection and intraoperative radiation therapy for recurrent endometrial cancer: technique and analysis of outcomes. Gynecol Oncol. 2006;101:280-6.

28. Arians N, Foerster R, Rom J, Roeder F, Debus J, Lindel K. Outcome of patients with local recurrent gynecologic malignances after resection combined with intraoperative electron radiation therapy (IOERT). Radiat Oncol. 2016;11:44

29. Yap OW, Kapp DS, Teng NN, Husain A. Intraoperative radiation therapy in recurrent ovarian cancer. Int J Radiat Oncol Biol Phys. 2005;63:1114-21.

30. Murray LJ, Lilley J, Hawkins MA, Henry AM, Dickinson P, Montefiore DS. Pelvic re-irradiation using stereotatic ablatibe radiotherapy (SABR): a systematic review. Radiother Oncol. 2017;125:231-22 Int J Radiat Oncol Biol Phys 2018;100:516-27.

31. Shaw EG, Gunderson LL, Martin JK, Beart RW, Nagorney DM, Podratz KC. Peripheral nerve and ureteral tolerance to intraoperative radiation therapy: clinical and dose-response analysis. Radiother Oncol. 1990;18:247-55.

32. Haddock MG, Miller RC, Nelson H. Combined modality therapy including intraoperative electron irradiation for locally recurrent colorectal cancer. Int J Radiat Oncol Biol Phys. 2011;79:143-50.

Ready to submit your research? Choose BMC and benefit from:

- fast, convenient online submission

- thorough peer review by experienced researchers in your field

- rapid publication on acceptance

- support for research data, including large and complex data types

- gold Open Access which fosters wider collaboration and increased citations

- maximum visibility for your research: over $100 \mathrm{M}$ website views per year

At $\mathrm{BMC}$, research is always in progress.

Learn more biomedcentral.com/submissions 